\title{
Efficient computation of the Nagaoka-Hayashi bound for multiparameter estimation with separable measurements
}

\author{
Lorcán O. Conlon $\mathbb{D i D}^{1 凶}$, Jun Suzuki (iD ${ }^{3 凶}$, Ping Koy Lam (iD) ${ }^{1,2}$ and Syed M. Assad ${ }^{1,2 凶}$
}

Finding the optimal attainable precisions in quantum multiparameter metrology is a non-trivial problem. One approach to tackling this problem involves the computation of bounds which impose limits on how accurately we can estimate certain physical quantities. One such bound is the Holevo Cramér-Rao bound on the trace of the mean squared error matrix. The Holevo bound is an asymptotically achievable bound when one allows for any measurement strategy, including collective measurements on many copies of the probe. In this work, we introduce a tighter bound for estimating multiple parameters simultaneously when performing separable measurements on a finite number of copies of the probe. This makes it more relevant in terms of experimental accessibility. We show that this bound can be efficiently computed by casting it as a semidefinite programme. We illustrate our bound with several examples of collective measurements on finite copies of the probe. These results have implications for the necessary requirements to saturate the Holevo bound.

npj Quantum Information (2021)7:110; https://doi.org/10.1038/s41534-021-00414-1

\section{INTRODUCTION}

Quantum mechanics simultaneously offers unique opportunities and limitations for metrology. On the one hand, uniquely quantum mechanical effects such as squeezing allow greater measurement sensitivity than is classically possible ${ }^{1,2}$. This is most evident in the search for gravitational waves, where the injection of squeezed light into LIGO has resulted in a significant increase in sensitivity ${ }^{3}$. Furthermore, quantum resources have been shown to offer enhanced measurement capabilities in a range of applications, including optical interferometry ${ }^{4-8}$, quantum superresolution $^{9,10}$, quantum-enhanced phase tracking ${ }^{11,12}$ and quantum positioning ${ }^{13,14}$ to name but a few examples. Fundamental limits to single-parameter measurement precisions can be computed using the quantum version of the Cramér-Rao bounds ${ }^{15-17}$. On the other hand, the uncertainty principle places fundamental limits on how well two or more non-commuting observables can be simultaneously measured ${ }^{18}$. Many of the applications of quantum estimation require the simultaneous measurement of multiple parameters ${ }^{19,20}$, which in general will not commute with each other. This means that a measurement that is optimal for one parameter may not be optimal for another which limits the precision with which we can measure them simultaneously ${ }^{21-25}$. Thus, in an effort to fully exploit quantum resources in real-world applications, there has been great experimental ${ }^{26-30}$ and theoretical interest in quantum multiparameter estimation ${ }^{31-43}$. Reviews of recent progress on the subject are given in refs. $24,25,40,44,45$.

Except for special cases involving qubits ${ }^{46}$ or estimating Gaussian amplitudes ${ }^{47,48}$, in general the problem of finding the optimal measurement that minimises the sum of the mean squared error (MSE) in multiparameter estimation is a non-trivial problem. Instead, one resorts to finding bounds on these errors ${ }^{49}$. Some of these bounds are the bounds based on the symmetric logarithmic derivatives $(S L D)^{15,16}$ and the right logarithmic derivatives $^{50}$ as well as the Gill-Massar ${ }^{51}$ bound. While these bounds are easy to compute, they are in general not tight.
A tighter bound for the sum of the MSE which can be achieved in the asymptotic limit is given by the Holevo Cramér-Rao bound ${ }^{52}$. The computation of the Holevo bound was recently cast as a semidefinite programme which has made it easy to compute. This was first performed for the Gaussian amplitude estimation problem $^{47}$ and was later generalised to an arbitrary model ${ }^{53}$. Furthermore, analytic expressions which upper and lower bound the Holevo bound have recently been found ${ }^{54}$. In some special cases, the measurement strategy required to reach the Holevo bound is known, for example, with pure state probes ${ }^{55}$ or for estimating a single parameter.

In general, the Holevo bound is only asymptotically achievable ${ }^{56-58}$, requiring a collective measurement over infinitely many copies of the probe state. A collective measurement here means that all copies of the probe state are measured simultaneously. In contrast, a separable measurement restricts the probe states to be measured individually. In practice, collective measurements are extremely challenging to perform and are not accessible to most experimental teams. Thus, it would be useful to have a tighter bound on the minimum achievable error when restricted to separable, single-copy measurements. One such bound for simultaneously estimating two parameters was introduced by Nagaoka ${ }^{59}$. This bound is at least just as tight as the Holevo bound and it can be saturated for probes in a two-dimensional Hilbert space ${ }^{60}$. However, just like the Holevo bound, Nagaoka's bound is not an explicit bound-it requires a further a non-trivial minimisation.

In this work, we generalise the Nagaoka bound to estimating more than two parameters, and we call this generalised bound the Nagaoka-Hayashi bound. This bound applies to separable measurements on a finite number of copies of the probe state, unlike the Holevo bound which, as mentioned above, is only asymptotically attainable in general. We further show that the minimisation required in the Nagaoka-Hayashi bound can computed using a semidefinite programme. This makes its

\footnotetext{
${ }^{1}$ Department of Quantum Science, Centre for Quantum Computation and Communication Technology, Australian National University, Canberra, ACT, Australia. ${ }^{2}$ School of Physical and Mathematical Sciences, Nanyang Technological University, Singapore, Singapore. ${ }^{3}$ Graduate School of Informatics and Engineering, The University of ElectroCommunications, Tokyo, Japan. ${ }^{\circledR}$ email: Iorcan.conlon@anu.edu.au; junsuzuki@uec.ac.jp; cqtsma@gmail.com
} 
computation accessible. We illustrate our results with two examples which highlight some of the interesting features of finite copy metrology which are inaccessible with conventional techniques. In both of these examples, we are able to find the positive operator valued measure (POVM) which saturates the bound, however whether this is always possible remains an open question.

\section{RESULTS}

Consider an $n$-parameter family of states $\left\{S_{\theta} \mid \theta \in \Theta \subseteq \mathbb{R}^{n}\right\}$ in a finite $d$ dimensional Hilbert space $\mathcal{H}_{q}$ with $\theta=\left(\bar{\theta}_{1}, \ldots, \theta_{n}\right)^{\top}$ denoting the $n$ independent true values that we wish to estimate. Let $\Pi=\left(\Pi_{1}, \ldots, \Pi_{M}\right)^{\top}$ be a column vector of $M$ POVM elements, where $(\cdot)^{\top}$ denotes partial transpose with respect to the classical subsystem. We require $\Pi_{m} \geq 0$ and $\Sigma_{m} \Pi_{m}=1$. Each outcome $m$ assigns an estimated value for $\theta_{j}$ through the classical estimator function $\hat{\theta}_{j m}$. The standard measure of estimation error when restricted to separable measurements is through the $n$-by- $n$ MSE matrix $V_{\theta}(\Pi, \hat{\theta})$ with entries

$$
\left[V_{\theta}(\Pi, \hat{\theta})\right]_{j k}=\sum_{m}\left(\hat{\theta}_{j m}-\theta_{j}\right)\left(\hat{\theta}_{k m}-\theta_{k}\right) \operatorname{Tr}\left[S_{\theta} \Pi_{m}\right], \quad \text { for } j, k=1, \ldots, n \text {. }
$$

The notation $\operatorname{Tr}[\cdot]$ in serif font is used to represent the trace of an operator in $\mathcal{H}_{q}$. For brevity of notation, hereafter we drop the argument and write the MSE matrix as $\mathrm{V}_{\theta}$. We aim to minimise the trace of the MSE matrix under the condition that our estimates are locally unbiased

$\sum_{m} \operatorname{Tr}\left[S_{\theta} \Pi_{m}\right] \hat{\theta}_{j m}=\theta_{j} \quad$ and $\quad \sum_{m} \frac{\partial}{\partial \theta_{k}} \operatorname{Tr}\left[S_{\theta} \Pi_{m}\right] \hat{\theta}_{j m}=\delta_{j k}$.

The Nagaoka bound for two-parameter estimation gives a lower bound on the trace of the MSE matrix as ${ }^{59}$

$$
\operatorname{Tr}\left[V_{\theta}\right] \geq \min _{X}\left\{\operatorname{Tr}\left[S_{\theta} X_{1} X_{1}+S_{\theta} X_{2} X_{2}\right]+\operatorname{TrAbs} S_{\theta}\left[X_{1}, X_{2}\right]\right\}=: c_{N},
$$

where $\operatorname{Tr}[\cdot]$ denotes the trace of a classical matrix in $\mathcal{H}_{c}$, an $n$ dimensional vector space, $\operatorname{Tr} A b s A$ is the sum of the absolute values of the eigenvalues of the operator $A$, and $X=$ $\left(X_{1}, X_{2}, \ldots, X_{n}\right)^{\top}$ is a vector of Hermitian estimator observables $X_{j}$ that satisfy the locally unbiased condition at $\theta$

$$
\operatorname{Tr}\left[S_{\theta} X_{j}\right]=\theta_{j} \quad \text { and } \quad \frac{\partial}{\partial \theta_{j}} \operatorname{Tr}\left[S_{\theta} X_{k}\right]=\delta_{j k}
$$

The Nagaoka bound was conjectured to be a tight bound for $\operatorname{Tr}\left[V_{\theta}\right]^{60}$.

\section{Computable multiparameter bound}

As we shall shortly prove, the Nagaoka bound can be generalised to more than two parameters. This result is stated as the following theorem.

Theorem 1 (Nagaoka-Hayashi bound). Let $\mathrm{V}_{\theta}$ be the MSE matrix of an unbiased estimate of $\theta$ for a separable measurement on a model $S_{\theta}$. Then, the trace of $V_{\theta}$ is bounded by

$$
\begin{aligned}
\operatorname{Tr}\left[V_{\theta}\right] \geq \min _{\mathbb{L}, X}\left\{\operatorname{Tr}\left[\mathbb{S}_{\theta} \mathbb{L}\right] \mid \mathbb{L}_{j k}=\mathbb{L}_{k j}\right. \text { Hermitian, } \\
\\
\left.\mathbb{L} \geq X X^{\top}, X_{j} \text { Hermitian satisfying }(4)\right\}=: c_{N H},
\end{aligned}
$$

where $\mathbb{S}_{\theta}=1_{n} \otimes S_{\theta}$ and $\mathbb{L}$ is an $n$-by- $n$ matrix of Hermitian operators $\mathbb{L}_{j k}$.

We use the symbol $\operatorname{Tr}[\cdot]$ to denote trace over both classical and quantum systems, i.e., over both $\mathcal{H}_{c}$ and $\mathcal{H}_{q}$. We call this bound the Nagaoka-Hayashi bound. However, the Nagaoka-Hayashi bound is not an explicit bound. Our second main result is that this bound, $c_{\mathrm{NH}}$ can be computed as a semidefinite programme.

\section{Related bounds}

Before proceeding on the proof and computation of the Nagaoka-Hayashi bound, we digress briefly to mention two related bounds. The first is the Holevo bound which can be written as $^{52}$

$$
\begin{aligned}
\operatorname{Tr}\left[V_{\theta}\right] \geq & \min _{\mathbb{L}, X}\left\{\operatorname{Tr}\left[\mathbb{S}_{\theta} \mathbb{L}\right] \mid \operatorname{Tr}\left[\mathbb{S}_{\theta} \mathbb{L}\right]\right. \text { real symmetric, } \\
& \left.\operatorname{Tr}\left[\mathbb{S}_{\theta} \mathbb{L}\right] \geq \operatorname{Tr}\left[\mathbb{S}_{\theta} X X^{\top}\right], X_{j} \text { Hermitian satisfying }(4)\right\}=: c_{H} .
\end{aligned}
$$

As mentioned before, the Holevo bound is a tight bound for collective measurements in the asymptotic limit. Since the conditions in the Nagaoka-Hayashi bound $\mathbb{L}_{j k}=\mathbb{L}_{k j}$ Hermitian implies $\operatorname{Tr}\left[\mathbb{S}_{\theta} \mathbb{L}\right]$ real symmetric and $\mathbb{L} \geq X X^{\top}$ implies $\operatorname{Tr}\left[\mathbb{S}_{\theta} \mathbb{L}\right] \geq \operatorname{Tr}\left[\mathbb{S}_{\theta} X X^{\top}\right]$, it is clear that the Nagaoka-Hayashi bound is more restrictive and hence is more informative compared to the Holevo bound. In other words $\mathrm{C}_{\mathrm{NH}} \geq \mathrm{C}_{\mathrm{H}}$.

The second related bound concerns estimation of physical observables. In this setting, the operators $X_{j}$ are given to us as Hermitian operators and the task is to estimate the expectation values $\operatorname{Tr}\left[S_{\theta} X_{j}\right]=x_{j}$. This situation is common, for example, in state tomography. Here, in place of the parameter-MSE matrix Eq. (1), we have the operator-MSE matrix

$$
\left[\tilde{U}_{\theta}(\Pi, \hat{x})\right]_{j k}=\sum_{m}\left(\hat{x}_{j m}-x_{j}\right)\left(\hat{x}_{k m}-x_{k}\right) \operatorname{Tr}\left[S_{\theta} \Pi_{m}\right], \quad \text { for } j, k=1,2, \ldots, n
$$

where we require the classical estimator $\hat{x}$ and POVM $\Pi$ to satisfy $\sum_{m} \hat{x}_{j m} \Pi_{m}=X_{j}$.

The derivatives of the state $S$ with respect to $\theta$ do not play any role here. A bound on the trace of $\tilde{U}_{\theta}$ is given by Hayashi's bound ${ }^{61}$

$\operatorname{Tr}\left[\tilde{U}_{\theta}\right] \geq \min _{\mathbb{L}}\left\{\operatorname{Tr}\left[\mathbb{S}_{\theta} \mathbb{L}\right]-\sum_{j} x_{j}^{2} \mid \mathbb{L}_{j k}=\mathbb{L}_{k j}\right.$ Hermitian, $\left.\mathbb{L} \geq X X^{\top}\right\}=: c_{N H-U}$.

As Hayashi's work is only available in Japanese, we summarise its main results in Supplementary Note 1 . If the given matrices $X$ happen to satisfy the locally unbiasedness condition Eq. (4) for $\theta$, then $\tilde{U}_{\theta}$ also forms a valid parameter-MSE matrix for those $\theta$. In this case, because of the additional restriction Eq. (8), it is clear that $c_{\mathrm{NH}-\mathrm{U}} \geq c_{\mathrm{NH}}$. Also in this setting, Watanabe et al. ${ }^{62}$ derived bounds for estimating two observables when restricted to certain classes of random and noisy measurements. In the case when both the observables and state $S$ are two-dimensional, these bounds are achievable. In fact, when the number of observables $n=2$, the minimisation over $\mathbb{L}_{L}$ can be performed analytically and $c_{\mathrm{NH}-\mathrm{U}}$ takes the explicit form ${ }^{61}$

$c_{\mathrm{NH}-\mathrm{U}}=\operatorname{Tr}\left[S_{\theta} X_{1} X_{1}+S_{\theta} X_{2} X_{2}\right]+\operatorname{TrAbs} S_{\theta}\left[X_{1}, X_{2}\right]-x_{1}^{2}-x_{2}^{2}$.

\section{Proof of main results}

In this section, we shall prove Theorem 1. To that end, we need to introduce some definitions. We rewrite the elements of the MSE matrix as

$$
\left[V_{\theta}\right]_{j k}=\operatorname{Tr}[S_{\theta} \underbrace{\sum_{m}\left(\hat{\theta}_{j m}-\theta_{j}\right) \Pi_{m}\left(\hat{\theta}_{k m}-\theta_{k}\right)}_{\left[\mathbb{L}_{\theta}\right]_{j k}}],
$$

where the MSE-matrix operator $\mathbb{L}_{\theta}(\Pi, \hat{\theta})$ is an $n$-by- $n$ matrix with operator elements. We introduce a classical matrix $\xi$ with elements 
$\xi_{j m}:=\hat{\theta}_{j m}-\theta_{j}$ so that

$$
\begin{aligned}
\mathbb{L}_{\theta} & =\sum_{m}\left(\begin{array}{lll}
\xi_{1 m} \Pi_{m} \xi_{1 m} & \xi_{1 m} \Pi_{m} \xi_{2 m} & \xi_{1 m} \Pi_{m} \xi_{3 m} \\
\xi_{2 m} \Pi_{m} \xi_{1 m} & \xi_{2 m} \Pi_{m} \xi_{2 m} & \xi_{2 m} \Pi_{m} \xi_{3 m} \\
\xi_{3 m} \Pi_{m} \xi_{1 m} & \xi_{3 m} \Pi_{m} \xi_{2 m} & \xi_{3 m} \Pi_{m} \xi_{3 m}
\end{array}\right) \\
& =\sum_{m}\left(\begin{array}{l}
\xi_{1 m} \\
\xi_{2 m} \\
\xi_{3 m}
\end{array}\right)\left(\xi_{1 m} \xi_{2 m} \xi_{3 m}\right) \otimes \Pi_{m},
\end{aligned}
$$

where we have set $n=3$ to simplify the presentation. The generalisation to arbitrary $n$ is straightforward. With this notation, it is clear that $\mathbb{L}_{\theta}$ is an operator on the extended Hilbert space $\mathcal{H}_{c} \otimes \mathcal{H}_{q}$. To anticipate the proof, it is useful to write $\mathbb{L}_{\theta}$ in the following form:

$$
\mathbb{L}_{\theta}=\left(\begin{array}{llll}
\Xi_{11} & \Xi_{12} & \ldots & \Xi_{1 M} \\
\Xi_{21} & \Xi_{22} & \ldots & \Xi_{2 M} \\
\Xi_{31} & \Xi_{32} & \ldots & \Xi_{3 M}
\end{array}\right)\left(\begin{array}{cccc}
\Pi_{1} & 0 & \ldots & 0 \\
0 & \Pi_{2} & \ldots & 0 \\
\vdots & \vdots & \ddots & \vdots \\
0 & 0 & \ldots & \Pi_{M}
\end{array}\right)\left(\begin{array}{ccc}
\Xi_{11} & \Xi_{21} & \Xi_{31} \\
\Xi_{12} & \Xi_{22} & \Xi_{32} \\
\vdots & \vdots & \vdots \\
\Xi_{1 M} & \Xi_{2 M} & \Xi_{3 M}
\end{array}\right),
$$

where $M$ is the number of POVM outcomes and $\Xi_{i j}=\xi_{i j} 1$. We can also introduce the following extension to $S_{\theta}, S_{\theta}=1 \otimes S_{\theta}$ so that the expression for the MSE matrix can be written as $V_{\theta}=\operatorname{Tr}\left[S_{\theta} \mathbb{L}_{\theta}\right]$. We are now ready to prove Theorem 1.

Proof. Suppose the optimal POVM and unbiased estimator have been found and are given by $\Pi$ and $X_{j}^{*}=$ $\sum_{m} \xi_{j m} \Pi_{m}, \quad$ for $\mathrm{j}=1, \ldots, \mathrm{n}$ which leads to the optimal MSE

$v^{*}=\sum_{j m} \xi_{j m}^{2} \operatorname{Tr}\left[S_{\theta} \Pi_{m}\right]=\operatorname{Tr}\left[\mathrm{S}_{\theta} \mathbb{L}_{\theta}^{*}\right]$.

We use asterisk to denote the optimal values and optimal operators. From $\Pi$ and $\hat{\theta}$, we can construct the estimator matrices $X_{j}^{*}=\sum_{m} \xi_{j m} \Pi_{m}$, for $\mathbf{j}=1, \ldots, \mathrm{n}$ so that

$\left(\begin{array}{l}X_{1}^{*} \\ X_{2}^{*} \\ X_{3}^{*}\end{array}\right)\left(X_{1}^{*} X_{2}^{*} X_{3}^{*}\right)=\left(\begin{array}{llll}\Xi_{11} & \Xi_{12} & \ldots & \Xi_{1 M} \\ \Xi_{21} & \Xi_{22} & \ldots & \Xi_{2 M} \\ \Xi_{31} & \Xi_{32} & \ldots & \Xi_{3 M}\end{array}\right)\left(\begin{array}{c}\Pi_{1} \\ \Pi_{2} \\ \vdots \\ \Pi_{M}\end{array}\right)\left(\Pi_{1} \Pi_{2} \ldots l \Pi_{M}\right)\left(\begin{array}{ccc}\Xi_{11} & \Xi_{21} & \Xi_{31} \\ \Xi_{12} & \Xi_{22} & \Xi_{32} \\ \vdots & \vdots & \vdots \\ \Xi_{1 M} & \Xi_{2 M} & \Xi_{3 M}\end{array}\right)$.

Comparing the above with Eq. (14) and using the result

$$
\left(\begin{array}{cccc}
\Pi_{1} & 0 & \ldots & 0 \\
0 & \Pi_{2} & \ldots & 0 \\
\vdots & \vdots & \ddots & \vdots \\
0 & 0 & \ldots & \Pi_{M}
\end{array}\right) \geq\left(\begin{array}{c}
\Pi_{1} \\
\Pi_{2} \\
\vdots \\
\Pi_{M}
\end{array}\right)\left(\begin{array}{llll}
\Pi_{1} & \Pi_{2} & \ldots & \Pi_{M}
\end{array}\right)
$$

which holds because $\Pi_{j}$ are positive operators that sums up to 1 (see Proposition II.9.1 of Holevo ${ }^{52}$ ), we arrive at $\mathbb{L}_{\theta}^{*} \geq X^{*} X^{* \top}$. With this, we can bound $v^{*}$ as

$$
\begin{aligned}
v^{*}= & \operatorname{Tr}\left[\mathbb{S}_{\theta} \mathbb{L}_{\theta}^{*}\right] \\
\geq & \min _{\mathbb{L}}\left\{\operatorname{Tr}\left[\mathbb{S}_{\theta} \mathbb{L}\right] \mid \mathbb{L}_{j k}=\mathbb{L}_{k j} \text { Hermitian, } \mathbb{L} \geq X^{*} X^{* \top}\right\} \\
\geq & \min _{\mathbb{L}, X}\left\{\operatorname{Tr}\left[\mathbb{S}_{\theta} \mathbb{L}\right] \mid \mathbb{L}_{j k}=\mathbb{L}_{k j} \text { Hermitian, } \mathbb{L} \geq X X^{\top},\right. \\
& \left.X_{j} \text { Hermitian satisfying }(4)\right\} \\
= & c_{N H} . \quad \square
\end{aligned}
$$

In the two-parameter case, we show in Supplementary Note 2 that $c_{N H}$ reduces to the original Nagaoka bound $c_{N}$ in Eq. (3). More generally, we are interested in minimising the weighted sum of the covariances which can be formalised with a positive weight matrix $W \geq 0$ and minimising $\operatorname{Tr}\left[W V_{\theta}\right]$. This problem can be handled by a suitable reparametrisation of the model which is presented in Supplementary Note 3.

The Nagaoka-Hayashi bound is not an explicit bound as it still requires a minimisation over $\mathbb{L}$ and $X$. Our next result concerns with the computation of this minimisation. Since $\mathbb{L}-X X^{\top}$ is the Schur's complement of the identity operator in $\left(\begin{array}{cc}\mathbb{L} & X \\ X^{\top} & 1\end{array}\right)$, the condition $\mathbb{L} \geq X X^{\top}$ is equivalent to $\left(\begin{array}{cc}\mathbb{L} & X \\ X^{\top} & 1\end{array}\right) \geq 0$. With this, $c_{\mathrm{NH}}$ can be written as the semidefinite programme

$$
\begin{gathered}
c_{\mathrm{NH}}=\min _{\mathbb{L}, X} \operatorname{Tr}\left[\mathbb{S}_{\theta} \mathbb{L}\right], \\
\text { subject to }\left(\begin{array}{cc}
\mathbb{L} & X \\
X^{\top} & 1
\end{array}\right) \geq 0
\end{gathered}
$$

where $\mathbb{L}_{j k}=\mathbb{L}_{k j}$ Hermitian and $X_{j}$ Hermitian satisfying the conditions Eq. (4) for local unbiasedness. The conversion to a standard semidefinite programme is performed in Supplementary Note 4. We also show in the same Supplementary Note that the worst case computational complexity for solving the SDP to an accuracy $\epsilon$ is $O\left((n d)^{3 / 2} \log (1 / \epsilon)\right)$.

The computation of the Holevo bound $c_{H}$ was shown to be a semidefinite programme by Albarelli et al. ${ }^{53}$. The difference between the Holevo bound and the Nagaoka-Hayashi bound is that in the former, the optimisation is performed directly on the covariance matrix $V=\operatorname{Tr}\left[\mathbb{S}_{\theta} \mathbb{L}\right]$, while in the latter the optimisation is performed on the operator $\mathbb{L}$. We note that both programmes can also be applied to compute the bound on the operator-MSE $C_{\mathrm{NH}-\mathrm{U}}$ Eq. (9) with little modification-the only changes needed are to replace the minimisation variables $X$ with the given observables and ignore the conditions Eq. (4).

In the following, we demonstrate our results by computing the Holevo and Nagaoka-Hayashi bounds for two illustrative examples-the estimation of orthogonal qubit rotations on the Bloch sphere in a phase damping channel and the simultaneous estimation of phase and loss in an interferometer. In the former, we find that the Holevo bound is always smaller than the Nagaoka-Hayashi bound, and in the latter we find that the two bounds are always equal. The minimisation problem was solved with the Yalmip toolbox ${ }^{63}$ for Matlab using the Mosek solver ${ }^{64}$.

Even though the semidefinite programme only returns numerical values for $X$ and $\mathbb{L}$, in some of these examples, the analytical forms for them can be inferred from the numerical solutions. Furthermore, every semidefinite programme Eq. (22) has a dual programme that involves performing a maximisation over the Lagrange multipliers associated with the primal programme ${ }^{65}$. That the inferred solutions are indeed optimal can then be verified by checking that the values for the primal and dual programmes coincide. For both of the examples considered, we present the dual solutions in Supplementary Note 7.

\section{Example 1: estimation of qubit rotations with a two-qubit probe}

Our first example concerns estimating the rotation experienced by qubit probes subject to the phase damping channel. This channel has particular relevance for modelling decoherence in trapped ions ${ }^{66-68}$. We consider the maximally entangled two-qubit state $(|01\rangle+|10\rangle) / \sqrt{2}$ as a probe. The first qubit acts as a signal-probe which passes through a channel imparting three small rotations: $\theta_{x}, \theta_{y}$ and $\theta_{z}$ about the $x, y$, and $z$ axes of the Bloch sphere. The rotated probe is then subject to the phase damping channel $\mathcal{E}$ with a known damping strength $\epsilon$

$\mathcal{E}[S]=\left(1-\frac{\epsilon}{2}\right) S+\frac{\epsilon}{2}\left(\sigma_{z} \otimes 1\right) S\left(\sigma_{z} \otimes 1\right)$. 
The second idler qubit is stored in a perfect quantum memory and remains unaffected by the rotation or phase damping. The resulting two-qubit state then has an approximate matrix representation in the computational basis as

$$
S_{\theta}=\frac{1}{4}\left(\begin{array}{cccc}
0 & -\mathrm{i} \theta_{x}-\theta_{y} & (1-\epsilon)\left(-\mathrm{i} \theta_{x}-\theta_{y}\right) & 0 \\
\mathrm{i} \theta_{x}-\theta_{y} & 2 & 2(1-\epsilon)\left(1-\mathrm{i} \theta_{z}\right) & (1-\epsilon)\left(\mathrm{i} \theta_{x}+\theta_{y}\right) \\
(1-\epsilon)\left(\mathrm{i} \theta_{x}-\theta_{y}\right) & 2(1-\epsilon)\left(1+\mathrm{i} \theta_{z}\right) & 2 & \mathrm{i} \theta_{x}+\theta_{y} \\
0 & (1-\epsilon)\left(-\mathrm{i} \theta_{x}+\theta_{y}\right) & -\mathrm{i} \theta_{x}+\theta_{y} & 0
\end{array}\right),
$$

which is valid to the first order in $\theta$. The partial derivatives of $S_{\theta}$ with respect to $\theta$ evaluated at $\theta=0$ are

$$
\begin{aligned}
& \frac{\partial S_{\theta}}{\partial \theta_{x}}=\frac{1}{4}\left(\begin{array}{cccc}
0 & -\mathrm{i} & -\mathrm{i}(1-\epsilon) & 0 \\
\mathrm{i} & 0 & 0 & \mathrm{i}(1-\epsilon) \\
\mathrm{i}(1-\epsilon) & 0 & 0 & \mathrm{i} \\
0 & -\mathrm{i}(1-\epsilon) & -\mathrm{i} & 0
\end{array}\right), \\
& \frac{\partial S_{\theta}}{\partial \theta_{y}}=\frac{1}{4}\left(\begin{array}{cccc}
0 & -1 & -(1-\epsilon) & 0 \\
-1 & 0 & 0 & (1-\epsilon) \\
-(1-\epsilon) & 0 & 0 & 1 \\
0 & (1-\epsilon) & 1 & 0
\end{array}\right) \quad \text { and } \\
& \frac{\partial S_{\theta}}{\partial \theta_{z}}=\frac{1}{2}\left(\begin{array}{cccc}
0 & 0 & 0 & 0 \\
0 & 0 & -i(1-\epsilon) & 0 \\
0 & \mathrm{i}(1-\epsilon) & 0 & 0 \\
0 & 0 & 0 & 0
\end{array}\right) \text {. }
\end{aligned}
$$

Single-parameter estimation. Let us start with the simple case when $\theta_{y}=\theta_{z}=0$ and we are only estimating the single parameter $\theta_{x}$. In a single-parameter estimation problem, the Holevo bound coincides with the Nagaoka-Hayashi bound and can always be saturated by a separable measurement. In this case, the two bounds can be achieved by the estimator operator

$X_{x}=\left(\begin{array}{cccc}0 & -\mathrm{i} & 0 & 0 \\ \mathrm{i} & 0 & 0 & 0 \\ 0 & 0 & 0 & \mathrm{i} \\ 0 & 0 & -\mathrm{i} & 0\end{array}\right)$

which gives $c_{\mathrm{H}, 1}=c_{\mathrm{NH}, 1}=1$, independent of $\epsilon$. The optimal measurement that saturates this bound is a projective measurement on the four orthogonal eigenvectors of $X_{x}$



This together with the estimation coefficients $\xi=(1,-1,-1,1)$ gives an estimation variance of $v_{x}=1$. The phase damping channel has no effect on the estimation precision.

Two-parameter estimation. Next, for estimating the two parameters $\theta_{x}$ and $\theta_{y}$ when $\theta_{z}=0$, the Holevo and Nagaoka bounds no longer coincide. The optimal matrices that achieve the minimum in the Holevo bound are found to be

$$
X_{x}=\left(\begin{array}{cccc}
0 & -\mathrm{i} & 0 & 0 \\
\mathrm{i} & 0 & 0 & 0 \\
0 & 0 & 0 & \mathrm{i} \\
0 & 0 & -\mathrm{i} & 0
\end{array}\right), \quad X_{y}=\left(\begin{array}{cccc}
0 & -1 & 0 & 0 \\
-1 & 0 & 0 & 0 \\
0 & 0 & 0 & 1 \\
0 & 0 & 1 & 0
\end{array}\right)
$$

which gives $C_{\mathrm{H}, 2}=2$. This means that there exists a sequence of collective measurements that can saturate a variance of $v_{x}=v_{y}=$ 1 in the asymptotic limit.

Unlike the single-parameter case, the optimal $X_{x}$ and $X_{y}$ operators for the Nagaoka bound are different from those which optimise the Holevo bound. For the Nagaoka bound the optimal matrices are

$X_{x}=\frac{1}{2-\epsilon}\left(\begin{array}{cccc}0 & -\mathrm{i} & -\mathrm{i} & 0 \\ \mathrm{i} & 0 & 0 & \mathrm{i} \\ \mathrm{i} & 0 & 0 & \mathrm{i} \\ 0 & -\mathrm{i} & -\mathrm{i} & 0\end{array}\right), \quad X_{y}=\frac{1}{2-\epsilon}\left(\begin{array}{cccc}0 & -1 & -1 & 0 \\ -1 & 0 & 0 & 1 \\ -1 & 0 & 0 & 1 \\ 0 & 1 & 1 & 0\end{array}\right)$

which gives $c_{\mathrm{NH}, 2}=4 /(2-\epsilon)$. Since there is a gap between the Holevo and Nagaoka bounds, a separable measurement cannot saturate the Holevo bound-a collective measurement is required. We show in Supplementary Note 5 that the Nagaoka bound is saturated by a family of five-outcome POVMs which gives $v_{x}=v_{y}=2 /(2-\epsilon)$. This means that when restricted to separable measurements, this is the smallest pair of variances possible.

Three parameter estimation. Finally, for estimating all three angles $\theta_{x}, \theta_{y}$, and $\theta_{z}$ simultaneously, we find the Holevo and Nagaoka-Hayashi bounds are

$c_{\mathrm{H}, 3}=2+\frac{1}{(1-\epsilon)^{2}} \quad$ and $\quad c_{\mathrm{NH}, 3}=\frac{4}{2-\epsilon}+\frac{1}{(1-\epsilon)^{2}}$.

Just like the two-parameter case, the gap between the two bounds implies that a collective measurement is required to saturate the Holevo bound. These bounds are achieved by the same estimator operators Eq. (28) for the Holevo bound and Eq. (29) for the Nagaoka-Hayashi bound with the additional

$X_{z}=\frac{1}{1-\epsilon}\left(\begin{array}{cccc}0 & 0 & 0 & 0 \\ 0 & 0 & -\mathrm{i} & 0 \\ 0 & \mathrm{i} & 0 & 0 \\ 0 & 0 & 0 & 0\end{array}\right)$.

We write down an explicit POVM that can approach $c_{\mathrm{NH}, 3}$ with $v_{x}=v_{y}=2 /(2-\epsilon)$ and $v_{z} \rightarrow 1 /(1-\epsilon)^{2}$ in Supplementary Note 5 showing that this bound is tight.

In order to quantify the estimation accuracy, we define the average preciseness for simultaneous estimation of $n$ parameters with $n /\left(v_{1}+\cdots+v_{n}\right)$ as a figure of merit on how good the estimators perform. By construction, a large average preciseness implies that all $n$ parameters can be determined accurately. We plot this quantity in Fig. 1 for all three estimation cases. We also note that in the two and three parameter examples, it is easy to check that the SLD Fisher information matrix is diagonal. Furthermore, the model is asymptotically classical and the Holevo bound coincides with the SLD bound ${ }^{21,69}$.

Estimation on multiple copies. We now demonstrate the usefulness of the Nagaoka-Hayashi bound and the associated SDP by examining the precision limits when we perform collective measurements on finite copies of the probe state. We denote the Nagaoka-Hayashi bound for $N$ copies of the same probe as $c_{\mathrm{NH}}\left(S_{\theta}^{\otimes N}\right)$. For a large number of copies of the probe state, we expect the Nagaoka-Hayashi bound to tend to the Holevo bound, $\lim _{N \rightarrow \infty} N c_{N H}\left(S_{\theta}^{\otimes N}\right)=c_{\mathrm{H}}$. For any finite $N$, we know that $N c_{N H}\left(S_{\theta}^{\otimes N}\right) \geq C_{H}$ which follows from $N c_{H}\left(S_{\theta}^{\otimes N}\right)=c_{H}$. Figure 2 shows how the gap between the two bounds shrinks for an increasing number of copies of the probe state. We consider up to three copies of the probe state. Without the Nagaoka-Hayashi bound, a brute force search for the optimal measurement strategy for three copies would require optimising an $M$ outcome POVM, where 
each outcome is a 64-by-64 matrix. Thus, the Nagaoka-Hayashi bound and the associated SDP offer an efficient way to investigate the asymptotic attainability of the Holevo bound. It provides a tool to address how fast optimal estimators on finite copies can converge to the asymptotic bound.

This example demonstrates several interesting features of finite copy metrology. First, we are able to definitively show that there exists a gap between the attainable precision with collective and separable measurements. Without a separable measurement bound, such a claim is not possible as any gap between a numerically optimal POVM and the Holevo bound may be a result of a deficiency in the numerical search as opposed to a physically meaningful gap. Second, as we are able to find a POVM which coincides with the Nagaoka-Hayashi bound, we are able to say with certainty that this POVM is optimal. Finally, we are able to investigate the attainability of the Holevo bound. While it is known that the Holevo bound is asymptotically attainable, it is not known how many copies of the probe state are required to get close to the Holevo bound. As mentioned above, to investigate this numerically with a POVM search is computationally very expensive. The SDP presented circumvents this and allows us to

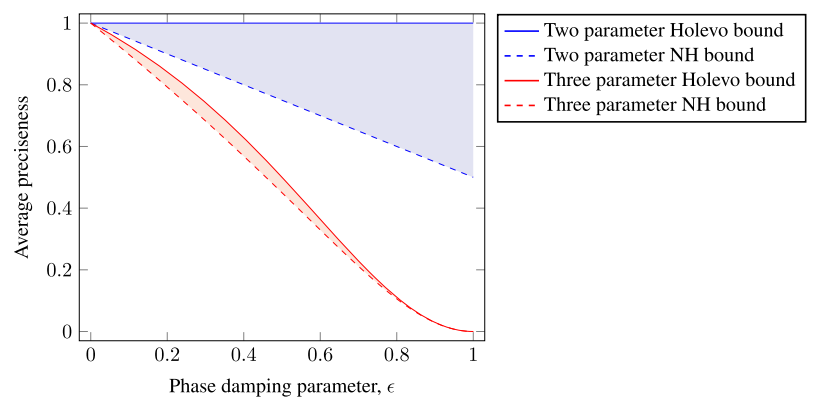

Fig. 1 Holevo and Nagaoka-Hayashi bounds for estimating multiple orthogonal rotation parameters simultaneously. For a maximally entangled two-qubit probe under the action of the phase damping channel, we plot the Holevo bounds (solid lines) and Nagaoka-Hayashi bounds (dashed lines) in terms of average preciseness for estimating two (blue) and three (red) orthogonal rotation parameters simultaneously. The Nagaoka-Hayashi bounds can be achieved by a separable measurement on a single probe, while the Holevo bound requires a collective measurement on a possibly infinite number of copies. The shaded area shows the gap between the two bounds. For estimating a single parameter, the Holevo and Nagaoka-Hayashi bounds coincide and are equal to the two-parameter Holevo bound. investigate the attainability of the Holevo bound in a numerically efficient manner.

\section{Example 2: Phase and transmissivity estimation in interferometry}

In our next example, we consider the problem of estimation of phase change $\phi$ and transmissivity $\eta$ in one arm of an interferometer as shown in Fig. 3. Following Crowley et al. ${ }^{33}$, we consider initial pure states with a definite photon-number $N$ across the two modes $\left|\psi_{\text {in }}\right\rangle=\sum_{k=0}^{N}|k, N-k\rangle a_{k}$, where $\left|N_{1}, N_{2}\right\rangle$ represents a state with $N_{1}$ photons in the first mode and $N_{2}$ photons in the second mode. One family of states with a fixed photon number is the Holland-Burnett states which are obtained by interfering two Fock states with an equal number of photons on a balanced beam splitter. These states lead to a phase estimation precision better than an interferometer driven by a coherent light source with the same number of photons ${ }^{70}$. The Holevo bound for the Holland-Burnett state was computed by Albarelli et al. ${ }^{53}$ for up to $N=14$. In general, the Holevo bound requires a collective measurement on several probes to be saturated. But for some values of $N$ and $\eta$, the Holevo bound can be saturated by a separable measurement, $\Pi^{(\phi)}$ that optimally measures the phase ${ }^{53}$.

We compute the Nagaoka bound for these states for different values of $\eta$ with $\phi=0$ for $N$ up to 14 using our SDP. We find that the Nagaoka and Holevo bounds always coincide (up to numerical noise). This is to be expected when $\Pi^{(\phi)}$ saturates the Holevo bound, but is not so obvious when it does not. The fact that there is no gap between the Holevo and Nagaoka bound implies one of the two possibilities: either (i) the Nagaoka bound is not tight or (ii) separable measurements are always optimal for simultaneous estimation of $\phi$ and $\eta$, in other words, collective measurements cannot do better. In the following, we show that the second statement is true.

Measurement saturating the Nagaoka bound. The initial pure state $\left|\psi_{\text {in }}\right\rangle=\sum_{k=0}^{N}|k, N-k\rangle a_{k}$ transforms in the lossy interferometer channel to the following state:

$S_{\phi, \eta}=\bigoplus_{l=0}^{N}\left|\psi_{l}\right\rangle p_{l}\left\langle\psi_{l}\right|$,

where each term in the direct sum

$\left|\psi_{l}\right\rangle=\sum_{k=1}^{N}|k-I, N-k\rangle a_{k} e^{i k \phi} \sqrt{\frac{b_{k l}}{p_{l}}}$
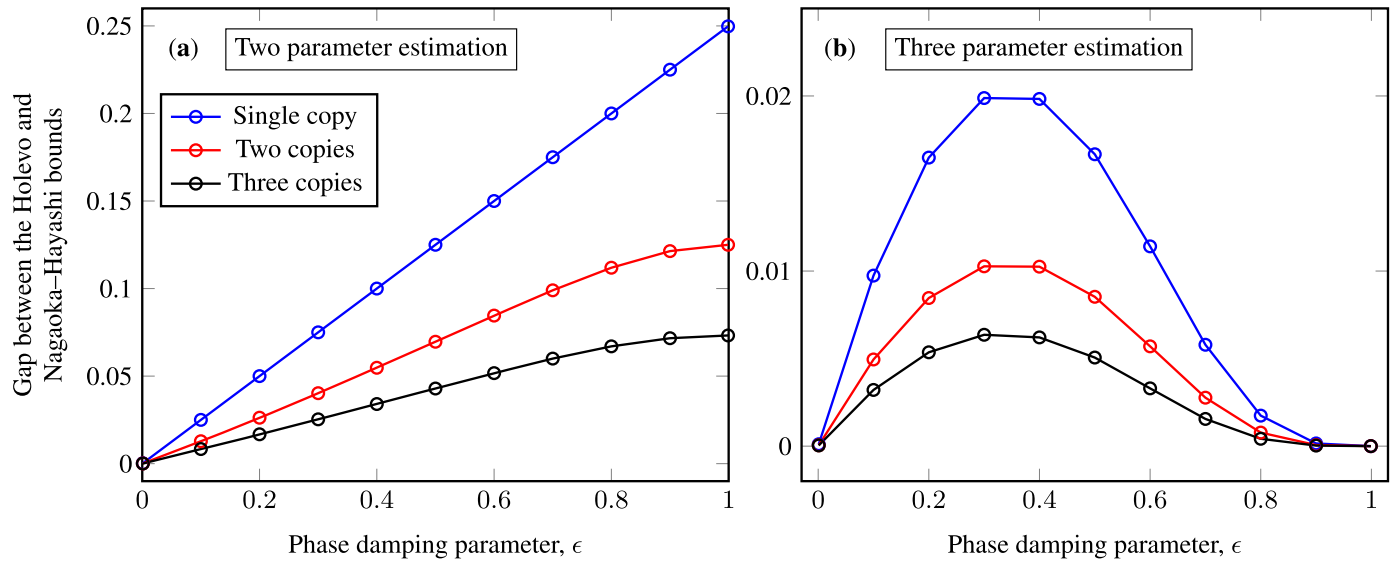

Fig. 2 Estimating multiple parameters with collective measurements on finite copies of the probe state. Both figures show how the gap between the Holevo and Nagaoka-Hayashi bounds shrinks as the number of copies of the probe state increases for estimating two (a) and three (b) parameters. The Nagaoka-Hayashi bounds are rescaled by the number of copies of the probe state to account for the resources used. 




Fig. 3 Schematic for optimal estimation of the phase shift $\phi$ and interferometer transmissivity $\eta$ using a two mode state $\left|\psi_{\text {in }}\right\rangle$ having definite photon-number $\boldsymbol{N}$. The measurement can be performed in two stages. The first stage (green block) involves performing a projective measurement over the photon-number subspace to determine the number of photons lost, $l$. The outcome of this measurement is then used to select a three-outcome POVM $\left\{\Pi^{()^{\prime}}\right\}$ for the second stage (black box). This measurement strategy saturates not only the Nagaoka bound, but also the Holevo bound.

represents a state with $/$ lost photons. The state $S_{\phi, \eta}$ is a mixed state with rank $N+1$. Here, $b_{k l}=\left(\begin{array}{c}k \\ l\end{array}\right) \eta^{k-l}(1-\eta)^{l}$ are the beamsplitter coefficients and $p_{l}$ represents the probability of losing $I$ photons. The partial derivatives of $S_{\phi, \eta}$ share the same direct sum structure

$$
\begin{aligned}
& \frac{\partial s_{\phi, \eta}}{\partial \phi}=\bigoplus_{l=0}^{N}\left(\left|\partial_{\phi} \psi_{l}\right\rangle p_{l}\left\langle\psi_{l}|+| \psi_{l}\right\rangle p_{l}\left\langle\partial_{\phi} \psi_{l}\right|\right), \\
& \frac{\partial s_{\phi, \eta}}{\partial \eta}=\bigoplus_{l=0}^{N}\left(\left|\psi_{l}\right\rangle \frac{\partial p_{l}}{\partial \eta}\left\langle\psi_{l}|+| \partial_{\eta} \psi_{l}\right\rangle p_{l}\left\langle\psi_{l}|+| \psi_{I}\right\rangle p_{l}\left\langle\partial_{\eta} \psi_{l}\right|\right)
\end{aligned}
$$

with each block having at most rank 2. Thus, what we have is a direct sum of pure state models, and for such a model, we have a separable measurement with a direct sum structure that can achieve the Holevo bound ${ }^{55}$. Each block can be measured separately but we cannot minimise $v_{\eta}+v_{\phi}$ separately in each block. This is because how much weight we attach to $\eta$ or $\phi$ in one block will depend on how much information about them that we can get from the other blocks. But regardless of the weights, each $I \neq N$ block requires at most a three outcome POVM to saturate the Holevo bound, so the total number of POVM outcomes needed is at most $3 N+1$. The extra 1 comes from the $I=N$ block where all photons are lost. An analytic POVM that saturates the Holevo bound for the $N=1$ case is given in Supplementary Note 6 . The dual solution to the Nagaoka-Hayashi bound is presented in Supplementary Note 7.

This problem demonstrates a very different but equally insightful feature of finite copy metrology compared to the qubit rotation problem. The simultaneous estimation of phase and loss has been very well studied in the literature ${ }^{6,8,33,53}$, however until now the fact that separable measurements are sufficient to reach the ultimate attainable precision had remained unknown. This insight was only possible with our SDP, which allowed the Nagaoka-Hayashi and Holevo bounds to be compared for large $N$. We plot the numerically calculated Nagaoka-Hayashi and Holevo bounds for different $N$ and $\eta$ in Fig. 4 . The fact that collective measurements are not required to reach the Holevo bound in this example may be important from a fundamental viewpoint.

\section{DISCUSSION}

We have presented the Nagaoka-Hayashi bound for the simultaneous estimation of multiple parameters when restricted to separable measurements. This ensures it is always tighter or just as tight as the Holevo bound. A gap between the two bounds would imply that the Holevo bound cannot be

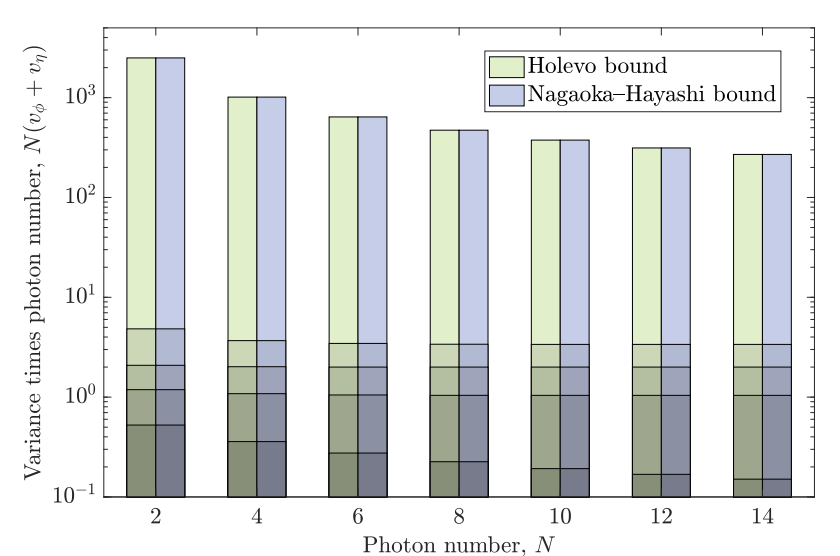

Fig. 4 The Holevo and Nagaoka-Hayashi bounds multiplied by photon number for estimating phase change $\phi$ and transmissivity $\eta$ using the Holland-Burnett states. The numerical results show that the two bounds coincide for $N$ up to and including 14 for any value of $\eta$. We show in the main text that there exists a separable measurement that reaches the ultimate attainable precisions in this example. In this case, a collective POVM cannot perform better than a separable POVM. Different shades correspond to different $\eta$ values, with darker colours corresponding to larger $\eta$. Results are shown for $\eta=0.01,0.25,0.5,0.75$, and 0.99 .

achieved with a separable measurement and a collective measurement is needed to saturate it. In addition, we have shown that the Nagaoka-Hayashi bound can be formulated as a semidefinite programme, allowing it to be solved efficiently. We have demonstrated our results with two examples. These examples illustrate how our results can be used to recognise when a collective measurement is essential and when it is not. Our results can be applied to many other problems in multiparameter quantum metrology and will help quantify the maximal advantage collective measurements have to offer. In some cases, a separable measurement is already optimal, simplifying any experimental realisation.

In the first example, we have assumed that the damping strength $\epsilon$ is known. However, in a practical setting, it would be more realistic to consider $\epsilon$ as a nuisance parameter, an unknown parameter that we are not interested in which nevertheless may hinder our measurement precision ${ }^{25,42,71}$. The quantum Cramér-Rao bound in the presence of nuisance parameters can be computed utilising a low-rank weight matrix ${ }^{25,71}$. As we show in Supplementary Note 3, our SDP formalism can be immediately 
applied to such cases. An interesting extension to this work would be to investigate examples which incorporate nuisance parameters.

\section{DATA AVAILABILITY}

The data that support the findings of this study are available from the corresponding author upon reasonable request.

\section{CODE AVAILABILITY}

The code that supports the findings of this study is available from the corresponding author upon reasonable request.

Received: 20 August 2020; Accepted: 14 April 2021; Published online: 15 July 2021

\section{REFERENCES}

1. Giovannetti, V., Lloyd, S. \& Maccone, L. Quantum-enhanced measurements: beating the standard quantum limit. Science 306, 1330-1336 (2004).

2. Giovannetti, V., Lloyd, S. \& Maccone, L. Advances in quantum metrology. Nat. Photonics 5, 222-229 (2011).

3. Aasi, J. et al. Enhanced sensitivity of the LIGO gravitational wave detector by using squeezed states of light. Nat. Photonics 7, 613-619 (2013).

4. Caves, C. M. Quantum-mechanical noise in an interferometer. Phys. Rev. D 23, 1693-1708 (1981).

5. Barnett, S. M., Fabre, C., \& Maitre, A. Ultimate quantum limits for resolution of beam displacements. Eur. Phys. J. D 22, 513-519 (2003).

6. Dorner, U. et al. Optimal quantum phase estimation. Phys. Rev. Lett. 102, 040403 (2009)

7. Kacprowicz, M., Demkowicz-Dobrzański, R., Wasilewski, W., Banaszek, K. \& Walmsley, I. A. Experimental quantum-enhanced estimation of a lossy phase shift. Nat. Photonics 4, 357-360 (2010).

8. Demkowicz-Dobrzański, R. et al. Quantum phase estimation with lossy interferometers. Phys. Rev. A 80, 013825 (2009).

9. Tsang, M., Nair, R. \& Lu, X. M. Quantum theory of superresolution for two incoherent optical point sources. Phys. Rev. X 6, 031033 (2016).

10. Tsang, M. Resolving starlight: a quantum perspective. Contemp. Phys. 60, 279-298 (2019).

11. Yonezawa, H. et al. Quantum-enhanced optical-phase tracking. Science 337, 1514-1517 (2012).

12. Zhang, L. et al. Quantum-limited fiber-optic phase tracking beyond $\pi$ range. Opt. Express 27, 2327-2334 (2019).

13. Giovannetti, V., Lloyd, S. \& Maccone, L. Quantum-enhanced positioning and clock synchronization. Nature 412, 417-419 (2001).

14. Lamine, B., Fabre, C. \& Treps, N. Quantum improvement of time transfer between remote clocks. Phys. Rev. Lett. 101, 123601 (2008).

15. Helstrom, C. W. The minimum variance of estimates in quantum signal detection. IEEE Trans. Inf. Theory 14, 234-242 (1968).

16. Helstrom, C. W. Minimum mean-squared error of estimates in quantum statistics. Phys. Lett. A 25, 101-102 (1967).

17. Belavkin, V. P. Generalized uncertainty relations and efficient measurements in quantum systems. Theor. Math. Phys. 26, 213-222 (1976).

18. Robertson, H. P. The uncertainty principle. Phys. Rev. 34, 163-164 (1929).

19. Cimini, V. et al. Quantum sensing for dynamical tracking of chemical processes. Phys. Rev. A 99, 053817 (2019).

20. Rehacek, J. et al. Multiparameter quantum metrology of incoherent point sources: towards realistic superresolution. Phys. Rev. A 96, 062107 (2017).

21. Ragy, S., Jarzyna, M. \& Demkowicz-Dobrzański, R. Compatibility in multiparameter quantum metrology. Phys. Rev. A 94, 052108 (2016).

22. Szczykulska, M., Baumgratz, T. \& Datta, A. Multi-parameter quantum metrology. Adv. Phys. $-X$ 1, 621-639 (2016).

23. Kull, I., Guérin, P. A. \& Verstraete, F. Uncertainty and trade-offs in quantum multiparameter estimation. J. Phys. A 53, 244001 (2020).

24. Demkowicz-Dobrzański, R., Górecki, W. \& Guță, M. Multi-parameter estimation beyond quantum Fisher information. J. Phys. A Math. Theor. 53, 363001 (2020).

25. Suzuki, J., Yang, Y. \& Hayashi, M. Quantum state estimation with nuisance parameters. J. Phys. A Math. Theor. 53, 453001 (2020).

26. Steinlechner, S. et al. Quantum-dense metrology. Nat. Photonics 7, 626-630 (2013).

27. Hou, Z., Zhu, H., Xiang, G. Y., Li, C. F. \& Guo, G. C. Achieving quantum precision limit in adaptive qubit state tomography. npj Quantum Inf. 2, 16001 (2016).

28. Roccia, E. et al. Entangling measurements for multiparameter estimation with two qubits. Quantum Sci. Technol. 3, 01 LT01 (2018)
29. Liu, Y. et al. Loss-tolerant quantum dense metrology with $\mathrm{SU}(1,1)$ interferometer Opt. Express 26, 27705-27715 (2018).

30. Hou, Z. et al. Deterministic realization of collective measurements via photonic quantum walks. Nat. Commun. 9, 1414 (2018).

31. Humphreys, P. C., Barbieri, M., Datta, A. \& Walmsley, I. A. Quantum enhanced multiple phase estimation. Phys. Rev. Lett. 111, 070403 (2013).

32. Genoni, M. G. et al. Optimal estimation of joint parameters in phase space. Phys. Rev. A 87, 012107 (2013)

33. Crowley, P., Datta, A., Barbieri, M. \& Walmsley, I. A. Tradeoff in simultaneous quantum-limited phase and loss estimation in interferometry. Phys. Rev. A 89 023845 (2014).

34. Gagatsos, C. N., Branford, D. \& Datta, A. Gaussian systems for quantum-enhanced multiple phase estimation. Phys. Rev. A 94, 042342 (2016).

35. Baumgratz, T. \& Datta, A. Quantum enhanced estimation of a multidimensional field. Phys. Rev. Lett. 116, 030801 (2016).

36. Chrostowski, A., Demkowicz-Dobrzański, R., Jarzyna, M. \& Banaszek, K. On superresolution imaging as a multiparameter estimation problem. Int. J Quantum Inf. 15, 1740005 (2017).

37. Pezzè, L. et al. Optimal measurements for simultaneous quantum estimation of multiple phases. Phys. Rev. Lett. 119, 130504 (2017).

38. Suzuki, J. Explicit formula for the Holevo bound for two-parameter qubit-state estimation problem. J. Math. Phys. 57, 042201 (2016).

39. Szczykulska, M., Baumgratz, T. \& Datta, A. Reaching for the quantum limits in the simultaneous estimation of phase and phase diffusion. Quantum Sci. Technol. 2 , 044004 (2017).

40. Albarelli, F., Barbieri, M., Genoni, M. G. \& Gianani, I. A perspective on multiparameter quantum metrology: from theoretical tools to applications in quantum imaging. Phys. Lett. A 384, 126311 (2020).

41. Assad, S. M. et al. Accessible precisions for estimating two conjugate parameters using Gaussian probes. Phys. Rev. Res. 2, 023182 (2020).

42. Tsang, M., Albarelli, F. \& Datta, A. Quantum semiparametric estimation. Phys. Rev. $X$ 10, 031023 (2020).

43. Carollo, A., Spagnolo, B., Dubkov, A. A. \& Valenti, D. On quantumness in multiparameter quantum estimation. J. Stat. Mech.: Theory Exp. 2019, 094010 (2019).

44. Sidhu, J. S. \& Kok, P. Geometric perspective on quantum parameter estimation. AVS Quantum Sci. 2, 014701 (2020).

45. Polino, E., Valeri, M., Spagnolo, N. \& Sciarrino, F. Photonic quantum metrology. AVS Quantum Sci. 2, 024703 (2020).

46. Suzuki, J. Parameter estimation of qubit states with unknown phase parameter. Int. J. Quantum Inf. 13, 1450044 (2015).

47. Bradshaw, M., Lam, P. K. \& Assad, S. M. Ultimate precision of joint quadrature parameter estimation with a Gaussian probe. Phys. Rev. A 97, 012106 (2018).

48. Bradshaw, M., Assad, S. M. \& Lam, P. K. A tight Cramér-Rao bound for joint parameter estimation with a pure two-mode squeezed probe. Phys. Lett. A 381, 2598-2607 (2017).

49. Hayashi, M. Asymptotic Theory of Quantum Statistical Inference: Selected Papers World Sci., 2005)

50. Yuen, H. \& Lax, M. Multiple-parameter quantum estimation and measurement of nonselfadjoint observables. IEEE Trans. Inf. Theory 19, 740-750 (1973).

51. Gill, R. D. \& Massar, S. State estimation for large ensembles. Phys. Rev. A 61 042312 (2000)

52. Holevo, A. S. Probabilistic and Statistical Aspects of Quantum Theory, Vol. 1 (Springer Sci. Bus. Media, 2011)

53. Albarelli, F., Friel, J. F. \& Datta, A. Evaluating the Holevo Cramér-Rao bound for multiparameter quantum metrology. Phys. Rev. Lett. 123, 200503 (2019).

54. Sidhu, J. S., Ouyang, Y., Campbell, E. T. \& Kok, P. Tight bounds on the simultaneous estimation of incompatible parameters. Phys. Rev. X 11, 011028 (2021).

55. Matsumoto, K. A new approach to the Cramér-Rao-type bound of the pure-state model. J. Phys. A: Math. Gen. 35, 3111-3123 (2002).

56. Kahn, J. \& Guță, M. Local asymptotic normality for finite dimensional quantum systems. Commun. Math. Phys. 289, 597-652 (2009).

57. Yamagata, K., Fujiwara, A. \& Gill, R. D. Quantum local asymptotic normality based on a new quantum likelihood ratio. Ann. Stat. 41, 2197-2217 (2013).

58. Yang, Y., Chiribella, G. \& Hayashi, M. Attaining the ultimate precision limit in quantum state estimation. Commun. Math. Phys. 368, 223-293 (2019).

59. Nagaoka, H. A new approach to Cramer-Rao bounds for quantum state estimation. In Asymptotic Theory of Quantum Statistical Inference: Selected Papers, 100-112 (World Sci. 2005). Originally published as IEICE Technical Report, 89, 228, IT 89-42, 9-14 (1989).

60. Nagaoka, H. A generalization of the simultaneous diagonalization of Hermitian matrices and its relation to quantum estimation theory. In Asymptotic Theory of Quantum Statistical Inference: Selected Papers, 133-149 (World Sci., 2005). Originally published as Trans. Jap. Soc. Indust. Appl. Math., 1, 43-56, (1991) in Japanese. Translated to English by Y. Tsuda. 
61. Hayashi, M. On simultaneous measurement of noncommutative observables. In Development of Infinite-Dimensional Non-Commutative Anaysis, 96-188 (Kyoto Univ., 1999).

62. Watanabe, Y., Sagawa, T. \& Ueda, M. Uncertainty relation revisited from quantum estimation theory. Phys. Rev. A 84, 042121 (2011).

63. Lofberg, J. Yalmip: a toolbox for modeling and optimization in matlab. in 2004 IEEE International Conference on Robotics and Automation (IEEE Cat. No. 04CH37508), 284-289 (IEEE, 2004).

64. ApS, M. The MOSEK Optimization Toolbox for MATLAB Manual. Version 9.0 http:// docs.mosek.com/9.0/toolbox/index.html (MOSEK ApS, 2019).

65. Boyd, S. \& Vandenberghe, L. Convex Optimization (Cambridge Univ. Press, 2004).

66. Huelga, S. F. et al. Improvement of frequency standards with quantum entanglement. Phys. Rev. Lett. 79, 3865-3868 (1997).

67. Myatt, C. J. et al. Decoherence of quantum superpositions through coupling to engineered reservoirs. Nature 403, 269-273 (2000).

68. Ma, J., Huang, Y.-X., Wang, X. \& Sun, C. P. Quantum Fisher information of the Greenberger-Horne-Zeilinger state in decoherence channels. Phys. Rev. A 84, 022302 (2011)

69. Suzuki, J. Information geometrical characterization of quantum statistical models in quantum estimation theory. Entropy 21, 703 (2019).

70. Holland, M. J. \& Burnett, K. Interferometric detection of optical phase shifts at the Heisenberg limit. Phys. Rev. Lett. 71, 1355-1358 (1993).

71. Suzuki, J. Nuisance parameter problem in quantum estimation theory: Tradeoff relation and qubit examples. J. Phys. A Math. Theor. 53, 264001 (2020).

\section{ACKNOWLEDGEMENTS}

This work was supported by the Australian Research Council (ARC) under the Centre of Excellence for Quantum Computation and Communication Technology (Grant No. CE170100012). J.S. was supported by the UEC Research Support Program, the University of Electro-Communications. The authors are grateful to Professor Nagaoka and Professor Hayashi for helpful discussions.

\section{AUTHOR CONTRIBUTIONS}

S.M.A. and J.S. conceived the project. S.M.A., J.S. and L.O.C. developed the theory, constructed the proof of Theorem 1, and worked out the optimal POVMs in the examples. L.O.C. and S.M.A. performed the numerical SDP simulations. L.O.C. and
S.M.A. wrote the manuscript. All authors contributed to discussions regarding the results in this paper. P.K.L. supervised the project.

\section{COMPETING INTERESTS}

The authors declare no competing interests.

\section{ADDITIONAL INFORMATION}

Supplementary information The online version contains supplementary material available at https://doi.org/10.1038/s41534-021-00414-1.

Correspondence and requests for materials should be addressed to L.O.C., J.S. or S.M.A.

Reprints and permission information is available at http://www.nature.com/ reprints

Publisher's note Springer Nature remains neutral with regard to jurisdictional claims in published maps and institutional affiliations.

(i) Open Access This article is licensed under a Creative Commons Attribution 4.0 International License, which permits use, sharing, adaptation, distribution and reproduction in any medium or format, as long as you give appropriate credit to the original author(s) and the source, provide a link to the Creative Commons license, and indicate if changes were made. The images or other third party material in this article are included in the article's Creative Commons license, unless indicated otherwise in a credit line to the material. If material is not included in the article's Creative Commons license and your intended use is not permitted by statutory regulation or exceeds the permitted use, you will need to obtain permission directly from the copyright holder. To view a copy of this license, visit http://creativecommons. org/licenses/by/4.0/.

(c) The Author(s) 2021 